\title{
Extension of Migdal-Watson formula and its application to binary breakup reaction
}

\author{
Riu Nakamoto, ${ }^{a, *}$ Makoto Ito, ${ }^{a}$ Akito Saito $^{b}$ and Susumu Shimoura ${ }^{c}$ \\ ${ }^{a}$ Department of Pure and Applied Physics, Kansai University, \\ Yamatecho 3-3-35, Suita, Japany \\ ${ }^{b}$ Department of Radiation Oncology, Hiroshima University Hospital, \\ 1-2-3 Kasumi, Minami, Hiroshima, Hiroshima, Japan \\ ${ }^{c}$ Center for Nuclear Study, School of Science, University of Tokyo, \\ 7-3-1, Hongo, Bunkyo-ku, Tokyo, Japan
}

E-mail: k298755@kansa-u.ac.jp, itomk@kansai-u.ac.jp

\begin{abstract}
New formula to describe a non-resonant background strength in binary breakup reactions is considered on the basis of the Migdal-Watson (MW) formula. The background strength is assumed to be generated by a direct breakup, which is an one-step transition from an initial bound state to final continuum states. The strength of the direct breakup is evaluated by the complex scaling method (CSM). We have found that the extended MW formula, which is proposed in the present study, nicely reproduces the strength of the direct breakup calculated by CSM. A relation of parameters newly introduced in the extended MW formula and a spatial size of the initial wave function is also discussed.
\end{abstract}

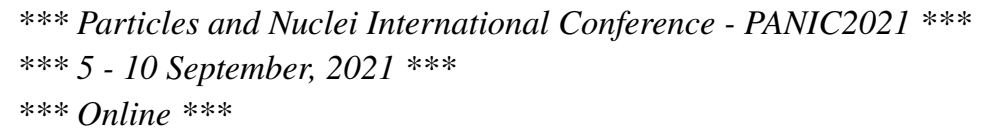

${ }^{*}$ Speaker 


\section{Introduction}

Inelastic scattering is a useful tool to explore the nuclear structure of the excited states of nuclei. In particular, the inelastic excitation of the nucleus to the continuum states above the particle decay threshold, which is usually called the breakup, is effective because we can clearly infer the intrinsic structure in the resonances by varying a combination of the emitted fragments. A typical and good example of such the breakup reaction going to the continuum can be seen in the breakup of ${ }^{12} \mathrm{Be}$ into the $\alpha+{ }^{8} \mathrm{He}$ and ${ }^{6} \mathrm{He}+{ }^{6} \mathrm{He}$ channels [1]. In this experiment, many resonant states having an intermediate width are identified by the careful multi-pole decomposition analysis.

Furthermore, the study of the resonant states by the breakup reaction is also important not only in the study of the nuclear structure but also in the study on the nucleosynthesis in cosmos because the reaction rate in the synthesis are strongly affected by the resonance parameters: resonance energy and decay width. In determining the resonance parameters, such as the resonance energies and the decay widths, the evaluation of the non-resonant background strength is indispensable because the resonant enhancements, which have a strong energy dependence, are embedded in the non-resonant background contribution. Therefore, it is quite important to consider the appropriate analytic function, which describes the non-resonant background contribution in the breakup reaction.

In this report, we propose a new formula to evaluate the non-resonant background contribution by extending the Migdal-Watson (MW) formula [2-4]. In constructing the analytic function, we assume that the non-resonant background strength is generated by the direct breakup process, which is the one step transition from the initial ground state to the final continuum state. In the calculation of the strength for the direct breakup, we employ the complex scaling method (CSM) [5], which is a powerful tool to describe the few-body continuum states. We check the validity of the extended MW formula by fitting the strength calculated from CSM. A set of the present results are compressed from Ref. [6].

\section{Theoretical framework}

\subsection{Complex scaling method}

In the present study, the direct breakup is defined by the one step transition from the initial ground state to the final excited state embedded in the continuum. The background strength generated by the direct breakup process is evaluated by the complex scaling method (CSM) [5]. In CSM, the transformation of the complex rotation with the rotation angle $\theta$

$$
\hat{U}(\theta) f(\mathbf{r})=e^{\frac{3}{2} i \theta} f\left(e^{i \theta} \mathbf{r}\right)=f^{\theta}
$$

is introduced for the arbitrary function of $f(\mathbf{r})$. Here the rotation on $\mathbf{r}$ should be read as the transformation on the radial part of the coordinate and hence, $r \rightarrow r e^{i \theta}$. The Schrödinger equation transformed by this complex rotation becomes

$$
\hat{H}^{\theta} \Psi^{\theta}=E^{\theta} \Psi^{\theta}
$$

where $\Psi^{\theta}$ is defined by Eq. (1) and $\hat{H}^{\theta}=\hat{U}(\theta) \hat{H} \hat{U}(\theta)^{-1}$. In the rotated Hamiltonian $\hat{H}_{\theta}$, the dynamical coordinates of $\mathbf{r}$ contained in $\hat{H}$ is complex rotated like $\hat{H}^{\theta}=\hat{H}\left(e^{i \theta} \mathbf{r}\right)$. The amplitude 
of the resonant wave function, which originally diverges in the asymptotic region, is damped in the large distant region by this complex rotation and hence, the usual computation technique for the bound state problem, such as the basis expansion method, is possible to apply. The energy eigenvalues calculated from the CSM plus basis expansion technique become the discrete and complex eigenvalue, $E^{\theta} \rightarrow E_{v}^{\theta}$ labeled by the eigenvalue number $v$. According to the ABC theorem [5], the energy eigenvalues for the bound state are invariant, and the energy eigenvalues for the resonances are clearly separated from the non-resonant continuum states in the complex energy plane [5].

The CSM is possible to apply to the calculation of the strength function, which represents the transition strength of the initial ground state $\left(\Psi_{i}\right)$ induced by the external field of $\hat{O}_{\lambda}$ with the multi-polarity $\lambda$. The definition of the strength function of $S_{\lambda}(E)$ is given by

$$
S_{\lambda}(E)=\sum_{f}\left|<\Psi_{f}\right| \hat{O}_{\lambda}\left|\Psi_{i}>\right|^{2} \delta\left(E-E_{f}\right)
$$

where $\Psi_{f}$ denotes the final state belonging to the $f$-th eigenstate excited by the external field of $\hat{O}_{\lambda}$. The strength function in Eq. (3) is calculated by a set of the solutions obtained by CSM, $\Psi_{i}^{\theta}, \Psi_{f}^{\theta}$ and $E^{\theta}$, according to the prescription in Ref. [5, 7, 8]. In the present study, the direct breakup of ${ }^{20} \mathrm{Ne}$ into $\alpha+{ }^{16} \mathrm{O}$ is considered because the ${ }^{20} \mathrm{Ne}$ is a typical example of the binary cluster system $[7,9]$. As for the external operator $\hat{O}_{\lambda}$, we consider the standard operator $\lambda=2$, such as

$$
\hat{O}_{\lambda=2}=\sqrt{4 \pi} \cdot R^{2} Y_{2,0}(\hat{\mathbf{R}})
$$

where $\mathbf{R}$ denotes the relative coordinate of the $\alpha$ and ${ }^{16} \mathrm{O}$ nuclei.

\subsection{Migdal-Watson theory and its extension}

In the s-wave breakup of the binary system composed of the charge neutral particles, the strength function can be expressed by the closed formula. This formula is called the Migdal-Watson (MW) theory [3, 4], which express the strength function in terms of the parameters of the effective range theory [10]. The detailed explanation of the MW formula has been shown in connection to the breakup of the di-neutron system in Ref. [2]. Here we explain the essence of the MW formula and extend the formula to the more general case of the binary breakup reaction.

In the MW theory, the monopole strength of the direct breakup with the relative energy $E$ for the binary fragments $\left(S_{\lambda=0}(E)\right)$ is given by

$$
S_{0}(E) \propto \frac{\sqrt{E}}{A E^{2}+B E+C},
$$

where the constants of $A, B$ and $C$ are given by the function of the scattering length and the effective range [2-4]. The MW formula shown in Eq. (5) is valid for the s-wave breakup from the initial wave function strongly localized inside of the nuclear interaction, which corresponds to the tightly binding system composed of the charge neutral fragments. Here we try to extend Eq. (5) so as to describe the direct breakup reaction of the general binary systems, which have the finite charge, the finite spin in the final scattering state $\left(S_{\lambda \neq 0}(E)\right)$ and the finite size of the initial wave function. 
We extend Eq. (5) to the following function:

$$
S_{\lambda}(E) \propto \frac{P_{\lambda}(k a) e^{-\beta E}}{A E^{2}+B E+C} .
$$

In this function, $P_{\lambda}(k a)$ denotes the penetration factor with the momentum $k$ for the binary decaying fragments and the channel radius $a$. $P_{\lambda}$ corresponds to the extension of the factor of $\sqrt{E}$ in Eq. (5). If we consider the limit of $k \rightarrow 0$ and $\lambda=0, P_{\lambda}(k a)$ is reduced to $\sqrt{E}$. On the contrary, the exponential function takes into account the tunneling effect of the initial wave function outside of the nuclear potential. The newly introduced parameters, such as $a$ and $\beta$, are sensitive to the spatial size of the initial wave function, which is not considered in the original MW formula. We try to reproduce the background strength generated by the direct breakup by controlling the five fitting parameters: $A, B, C, a, \beta$.

\section{Results}

The fitting result using the extended MW formula in Eq. (6) is shown in Fig. 1. The quadrupole strength for ${ }^{20} \mathrm{Ne} \rightarrow \alpha+{ }^{16} \mathrm{O}$ are shown. The strength function calculated by the CSM (solid curve with dots) is nicely reproduced by the extended MW formula (open circles). The parameters used in the extended MW formula in Eq. (6) are $A=0.025, B=-0.21, C=0.48, a=5.5 \mathrm{fm}$ and $\beta=$ $0.015 \mathrm{MeV}^{-1}$. In this figure, the open square with the error bar means the resonance energy with the decay width of the potential resonance in the final state. The resonant state in the final continuum state exists around the peak position in the strength function.

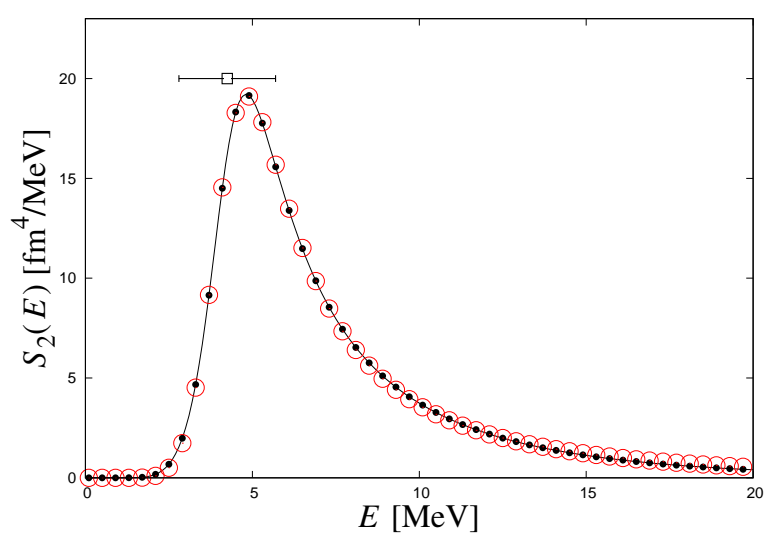

Figure 1: Comparison of the strength function of ${ }^{20} \mathrm{Ne} \rightarrow \alpha+{ }^{16} \mathrm{O}$ with fitting results by the extended Migdal-Watson formula in Eq. (6). The $\alpha$ threshold energy is set to the zero point in the abscissa. The solid curve and the open circles represent the strength function calculated from CSM and fitting results by Eq. (6), respectively. The open square with the error bar shows the resonance energy with the decay width. This figure is taken from Ref. [6].

We check the sensitivity of the parameters $\beta$ and $a$ to the spatial size of the initial wave function by controlling the binding energy of the initial wave function. As the initial wave function becomes 
the weakly bound state, and the resultant size of the wave function is more extended, the parameter of $\beta$ and $a$ become large. Thus, these two parameters, which are introduced in extending the MW formula, reveals the sensitivity to the spatial size of the initial wave function.

\section{Summary}

In summary, we have considered the analytic function to evaluate the non-resonant background strength in the binary breakup reaction. Here the background strength is assumed to be generated by the direct breakup, which is the one step transition from the initial ground state to the final continuum state. The analytic function is constructed by extending the Migdal-Watson (MW) formula $[2-4,10]$. The MW formula gives the function of the quadratic energy denominator with the numerator of the square root of energy, such as $\sqrt{E} /\left(A E^{2}+B E+C\right)$.

We have improved the MW formula so as to include the effects of the finite spins, the finite charge and the finite size of the initial wave function. First, the numerator of $\sqrt{E}$ is replaced by the penetration factor of $P_{\lambda}(k a)$ to take into account the effects of the finite spin $(\lambda)$ and finite charge. Furthermore, we have introduced the exponential damping factor, $\exp (-\beta E)$, which is originated from the tunneling tail of the initial wave function outside of the nuclear interaction.

We have checked the validity of the extended MW formula by fitting the strength function obtained from the theoretical calculation of the direct breakup. In the present analysis, we have calculated the background strength in the breakup of ${ }^{20} \mathrm{Ne} \rightarrow \alpha+{ }^{16} \mathrm{O}$ [7] on the basis of the complex scaling method (CSM) [5]. The CSM strength function is nicely reproduced by the extended MW formula. Moreover, we have demonstrated that the newly introduced parameters, suhc as $\beta$ and $a$, are sensitive to the spatial size of the initial wave function. Since we have confirmed the availability of the MW formula in the binary breakup, it is important to apply the formula to other binary breakup reactions, such as ${ }^{12} \mathrm{Be} \rightarrow \alpha+{ }^{8} \mathrm{He}$ [1]. The application to ${ }^{12} \mathrm{Be}$ is now under progress.

\section{References}

[1] A. Saito et al., Mod. Phys. Lett. A, Vol. 25, No. 21-23, 2010, pp. 1858-1861.

[2] S. Shimoura, Eur. Phys. Jour. Plus 133, 2018, 463.

[3] A. Migdal, Sov. Phys. JETP 1, 1955, 2.

[4] K. Watson, Phys. Rev. C88, 1952, 1163.

[5] S. Aoyama, T. Myo, K. Katō and K. Ikeda, Prog. Theor. Phys. 116, 2006, and references therein, pp.1-35.

[6] R. Nakamoto, M. Ito, A. Saito and S. Shimoura, Phys. Rev. C104, 034602, (2021).

[7] A. T. Kruppa and K. Katō, Prog. Theor. Phys. 84, 1990, 1145.

[8] T. Myo, A. Ohnishi and K. Katō, Prog. Theor. Phys. 99, 1998, 801.

[9] H. Horiuchi et al., Prog. Theor. Phys. Suppl. 2012 Jan; 192: 1-238, and references therein.

[10] N. Austern, Nucl. Phys. 7, 1958, 195. 